\title{
Multi-Rate, Dynamic and Compliant Region of Interest Coding for JPEG2000
}

\author{
Jun Hou, Xiangzhong Fang, Jiliang Li and Haibin Yin,Songyu Yu
}

\author{
Shanghai Jiaotong university, Shanghai 200030, China
}

\begin{abstract}
A method is proposed to encode multiple regions of interest(ROI) in JPEG2000 image. It rearranges truncation point for every codeblock in each layer. It assigns higher bitrate to ROI and lower bitrate to non-ROI and combines them to codestream. The proposed strategy produces a fully compliant JPEG2000 codestream. It allows transmission of different ROIs with different priorities and supports dynamic delineation and prioritization of them. Experimental results demonstrating the validity of the proposed approach are presented.
\end{abstract}

\section{INTRODUCTION}

JPEG2000 standard[1] for still images is intended to overcome the shortcomings of the existing JPEG standard. It employs the discrete wavelet transform, and incorporate functionality such as lossless and lossy compression spatial and quality scalability, region of interest coding, random code-stream access and error-resilient coding. The encoding of ROIs in an image with quality better than the background is one of the new features of the JPEG2000.

Several techniques have been proposed for ROI-based image coding. JPEG2000 currently supports two methods to encode ROIs: the general scaling based method(GSBM)[2] and the maximum shift(MAXSHIFT) method [3]. In GSBM, the coefficients associated with an ROI are scaled up so that the corresponding bits are placed in higher bit-planes. Given the hierarchical structure of the JPEG2000 codestream, these scaled-up bit-planes are placed in the final bitstream before any bit-planes associated with the background. Depending on the scaling value, some bits of the ROI coefficients may be encoded along with background coefficients. At the decoder side, the ROI is decoded first before the rest of the image. The main disadvantage of GSBM is that it needs to encode and transmit the shape information of an ROI, resulting in an increase in the computational complexity as well as the bitrate. Only rectangle and ellipse ROI shapes are allowed. The MAXSHIFT method scales up the coefficients associated with an ROI well above the background and eliminates the need of generating the mask at the encoder. At the decoder, the nonzero ROI and background coefficients are identified by their magnitude. There is no need to explicitly transmit the shape information of the ROI. The main drawback of the MAXSHIFT method is that it is not possible to define the relative importance of the ROI and background coefficients. Also, different ROIs cannot have their own scaling value.

A flexible method called bitplane-by-bitplane shift (BbBShift)to define ROIs is proposed in [4]. It supports arbitrary ROI shapes and scaling values. Instead of shifting the bitplanes all at once by the same scaling value, it shifts them on a bitplane-by-bitplane basis. The method allows for the decoding of background information once a high enough quality ROI is achieved. A similar method called partial significant bit-planes shift(PSBShift) was proposed in [5]. It supports arbitrary shaped ROI coding without coding the shape and enables the flexible adjustment of compression quality in the ROI and the background. Both the above methods are not fully compatible with the current JPEG2000 standard.

A method called prioritized ROI coding is proposed in [6]. It creates several priority levels according to the distance of a region from the ROI. This particular prioritization method employs a foveated image and allows gradual increase in peripheral quality loss. It can encode multiple ROI with different priorities. The relative importance of ROIs is static and cannot be changed during encoding. A method of rearranging packets is proposed in [7]. It shifts ROI packets to initial layers to make possible the decoding of ROIs before the background. It allows dynamic and flexible definition of ROI. The above methods increase final bitrate due to null packets. Weighting the packets in ROI and resequencing the layer, [9] browses the JPEG2000 image with minimum ROI distortion in each layer. In [10] and [11], implicit method is proposed. By weighting the distortion of those CBs whose samples contribute to ROI, it assigned a higher priority to ROI. It allows for coding multiple ROI coding with different priority.

In this paper, we propose a ROI coding technique where background information is encoded along with the ROIs. It is dynamic ROI scheme that supports the definition and handling arbitrary-shaped ROIs, dynamic definition and prioritization of new and existing ROIs and creation of compliant datastream.

The paper is organized as follows. The method is 


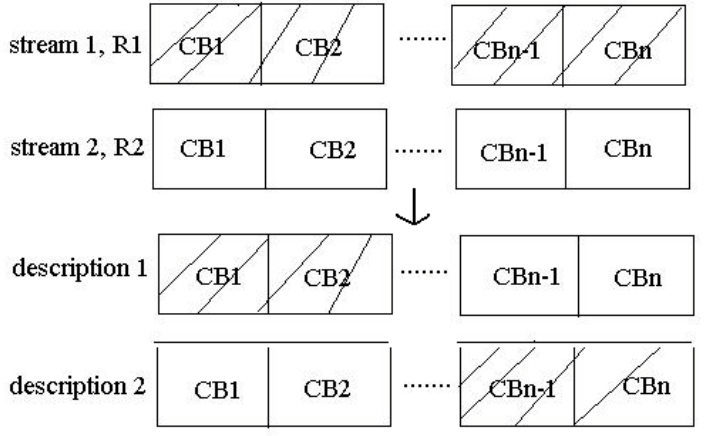

Fig.1 illustration of MDC [8]

proposed in Section 2. Section 3 presents the simulation results. Finally, concluding remarks are given in section 4.

\section{PROPOSED ALGORITHM}

The proposed algorithm is motivated by multiple description coding (MDC) schemes for JPEG2000 [8]. In MDC approach, two or more non hierarchical independently decodable representations of the same date, or descriptions are generated. If a single description is received, the side decoder can reconstruct the data with lower quality. If all descriptions are received, the data are recovered with lower distortion. In [8], a MDC method is proposed for JPEG2000. It generates two streams at different rate R1 and R2 respectively. For each layer of either bitstream, the CBs are grouped into two sets having similar rate-distortion character. One description is obtained by properly combining CBs of SET1 from the first stream, with CBs of SET2 from the second stream. The other description is built by taking CBs of SET2 in the first stream and those of SET1 in the second stream. In a single description, some of CBs get definition quality(encoded at R1 bitrate), others have lower quality(encoded at R2 bitrate). The illustration of [8] is showed in Fig.1.

The above MDC gives us a hint that in each layer, we can truncate ROI CBs and BG CBs at different target bitrate and combine them into packets. In each layer, we assigned high bitrate to ROI and low bitrate to BG dynamically. Therefore, the ROI has higher quality than BG in same layer. The above idea can be used for single ROI coding and multiple ROI coding. The details of proposed scheme are described as follows.

\section{A. Single ROI Coding}

step 1).The image is encoded at bit-rate $R 1$ with $L$ levels and $N$ layers. The target bit-rate for each layer are: $R_{11}, R_{12}, \ldots . R_{1 N}$, separately. Record each ROI CB's truncation point at each layer.

step 2) The image is encoded at bit-rate Rb with L levels and $N$ layers. The target bit-rate for each layer are: $R_{b 1}, R_{b 2}, \ldots \ldots . R_{b N}$ separately. For each layer, there is
$R_{1 i} \geq R_{b i}(i=1 \ldots N)$. Record each BG CB's truncation point. The relation of $R_{1 i}$ and $R_{b i}$ determines the relative importance of the ROI in the image. The greater the number of quality layers $N$, the better is the resolution scalability. step 3) The CBs describing an ROI across the different levels are identified. The result packet is obtained by properly combining ROI CBs streams truncated at bitrate $R 1$ with those of $B G C B$ s corresponding to $R b$.

Note that the size of each CB must be same in step 1) and step 2). The target rate $R_{1 i}$ and $R_{b i}$ that determine the priority of ROI can be allocated freely. For instance, we can allocate the budget rate according to foveated inspection-the quality difference between ROI and BG becomes smaller with the bitrate increasing. Let

$$
\Delta B_{i}=R_{1 i}-R_{b i}
$$

There are:

$$
\Delta B_{1} \geq \Delta B_{2} \geq \ldots . \Delta B_{N} \geq 0
$$

Fig.2 illustrates the proposed algorithm. The dark blocks present streams of ROI CBs. Other blocks illustrate those BG CBs. In each layer, the truncation points of ROI CBs are decided at given rate $R_{1 i}$ (see Fig.2(a)). The truncation point of $\mathrm{BG}$ are decided at given rate $R_{b i}$ (see Fig.2(b)). The result codestream combines them as Fig.2(c) shows.

\section{B. Multiple ROI Coding}

Multiple ROI coding requires multiple ROIs to be coded

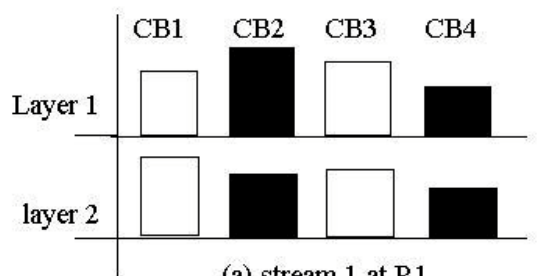

(a) stream 1 at $\mathrm{R} 1$

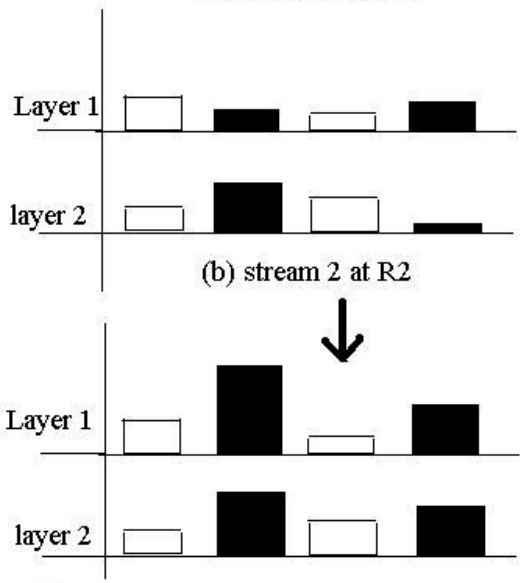

(c) final combined stream with ROI coding

Fig.2 dynamic CBs combination using JPEG2000 coding. Dark CBs illustrate ROI CB. 
with different quality according to their priorities in an image. The proposed method supports efficient multiple ROI coding by truncating multiple ROI CBs at different target bitrate in each layer. The priorities of those ROIs can change dynamically at coding time.

Suppose there are M ROIs to be encoded with different priorities. $\mathrm{L}$ and $\mathrm{N}$ denote the level and the layer in coding, respectively. In $j^{\text {th }}(\mathrm{j}=1 \ldots . . \mathrm{N})$ layer, we decide each CBs truncation point corresponding to bitrate $R_{m j}(m=1 \ldots . M)$ and $R_{b j}$, separately. $R_{m j}$ denotes the budget bitrate of $m^{t h}$ ROI in $j^{\text {th }}$ layer. $R_{b j}$ is the target bitrate of BG in $j^{\text {th }}$ layer. In each layer, the bit-rate $R_{m j}$ assigned to the $m^{\text {th }}$ ROI determines the relative importance of the region. If a CB belongs to several ROIs, its truncation point in $j^{\text {th }}$ layer correspond to the maximum bitrate assigned to these ROIs:

$$
R_{j}=\max \left\{R_{m j}, m \in(\text { ROIs that the CB belongs })\right\}
$$

The result stream is obtained by properly combining encoded streams of $m^{\text {th }}$ ROI CBs coded at $R_{m j}$ and that of BG CBs coded at $R_{b j}$.

\section{Complexity}

The proposed method may increase the computing cost compared to normal JPEG2000 without ROI coding since it applies PRCRopt more times. There are several cases to be discussed. Suppose there are N layers and M ROIs to be coded.

case 1): For the $m^{\text {th }}$ ROI, its target bit is $R_{m j}(\mathrm{j}=1 \ldots \mathrm{N})$ in $j^{\text {th }}$ layer. The ROI-budget-bitrate set is:

$\mathfrak{R}_{R O I}=\left\{R_{m j}, j=1 . . N, m=1 \ldots M\right\}$,

For the BG, its budget bitrate set is:

$$
\begin{aligned}
\mathrm{B} & =\left\{R_{b j}, j=1 \ldots N\right\} . \\
& \forall R_{m j} \in \mathfrak{R}_{R O I}, \text { there is } R_{m j} \in \mathrm{B} . \text { For every } \mathrm{CB} \text {, the }
\end{aligned}
$$

proposal scheme decides truncation point once in each layer. In some inspection, the method is similar as [7] that rearrange the packet in layers.

case 2): In the general case, there is $R_{m j}$ that satisfies $R_{m j} \notin \mathrm{B}$. We have to decide truncation point for ROI and BG independently in each layer. PCRDopt is applied more than one times. In extreme cases, for any $R_{m j}$, there is $R_{m j} \notin \mathrm{B}$. As a result, we apply PCRDopt $(\mathrm{M}+1)$ times in each layer, compared to normal JPEG2000 coding.

\section{Compatibility and Functionality}

The proposed method produces codestream compatible with JPEG2000 standard ones. Any JPEG2000 decoder can decode the final codestream.

The proposed method offers a lot of advantages. It can encode multiple ROIs and include background information along with them. It is possible for each ROI to have its own priority level. Compared to the way of implicit method [10][11], it allows flexible and dynamic ROI handling in each layer. During creating of a new layer all current demands like new ROI definition or changing prioritization of ROI, can be considered. For instance, there are two ROIs. At low bitrate, the priority of first ROI is higher, so we can set $R_{1 i}>R_{2 i} \geq R_{b i}, \mathrm{i}=1 \ldots \mathrm{n}$. At higher bitrate, we pay more attention to the second ROI. Thus we only set $R_{b i \leq} R_{1 i}<R_{2 i}$, $\mathrm{i}=\mathrm{n}+1 \ldots \mathrm{N}$.

The method does not change image coding information. If the image is encoded losslessly, the final datastream does not change this property.

\section{SIMULATIONS}

The performance of proposal method is evaluated with a $2048 \times 1536$ gray image using 5 levels and 15 layers to compress. The target bitrate is $0.125 \mathrm{bpp}$ (bit per pixel). Fig.3 show the result of multiple ROIs coding. In the $1^{\text {st }}$ layer, only one ROI has bitrate a little higher than BG. In each layer, it has bitrate 1.5 times as BG till it reaches the target bitrate. So only one ROI is shown in the $1^{\text {st }}$ layer as Fig.3(a) shows. In the $2^{\text {nd }}$ layers, we define the $2^{\text {nd }}$ ROI that has a bitrate 2 times as BG in the following layers till it reaches the budget bitrate: 0.125bpp. Fig.3(b) shows the coding result in $5^{\text {th }}$ layer. The $3^{\text {rd }}$ ROI has the same bitrate as BG until the $7^{\text {th }}$ layer. From the $7^{\text {th }}$ layer, the $3^{\text {rd }}$ ROI has a target bitrate 8 times as that of $B G$ till it reaches the target bitrate. Its quality is increased rapidly. It has highest priority among three ROIs. The result in $7^{\text {th }}$ layer is shown in Fig.3(c). In the last layer, all ROIs and BGs have achieved the same target bitrate: 0.125bpp as Fig.3(d) illustrates.

\section{CONCLUSION}

In this paper, we propose a new method for the incorporation of ROI into JPEG2000. The proposed method allows dynamic and flexible definition of regular ROIs. It is realized by assigning different target bitrate to ROIs and BGs in each quality layer and combining to the packets. The produced codestream is compatible with standard.

\section{REFERENCES}




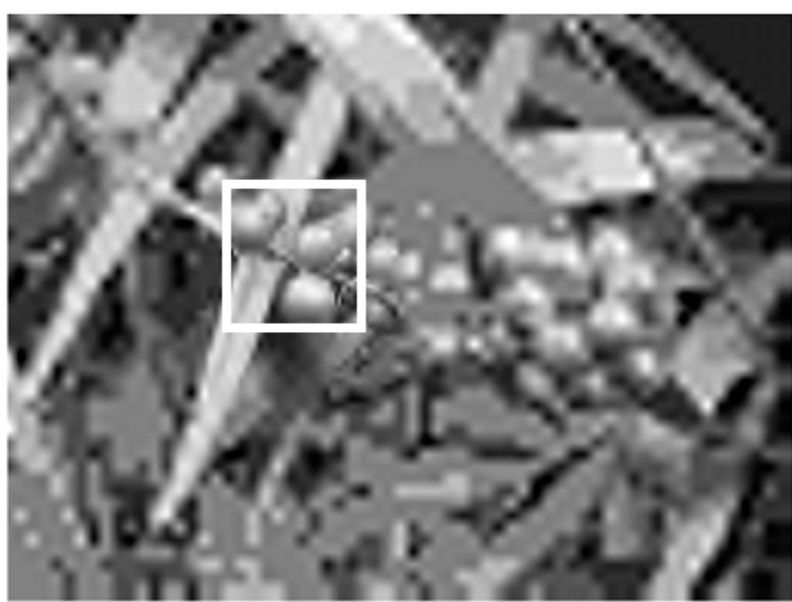

(a)

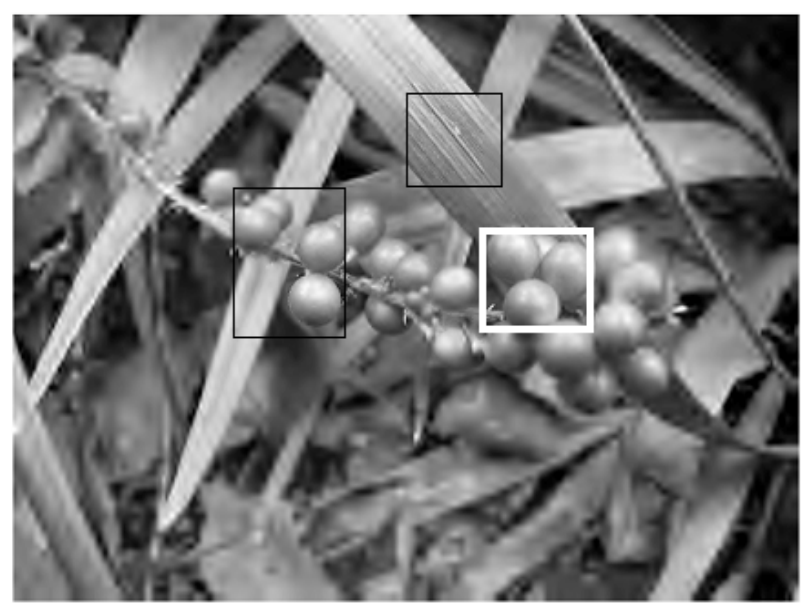

(c)

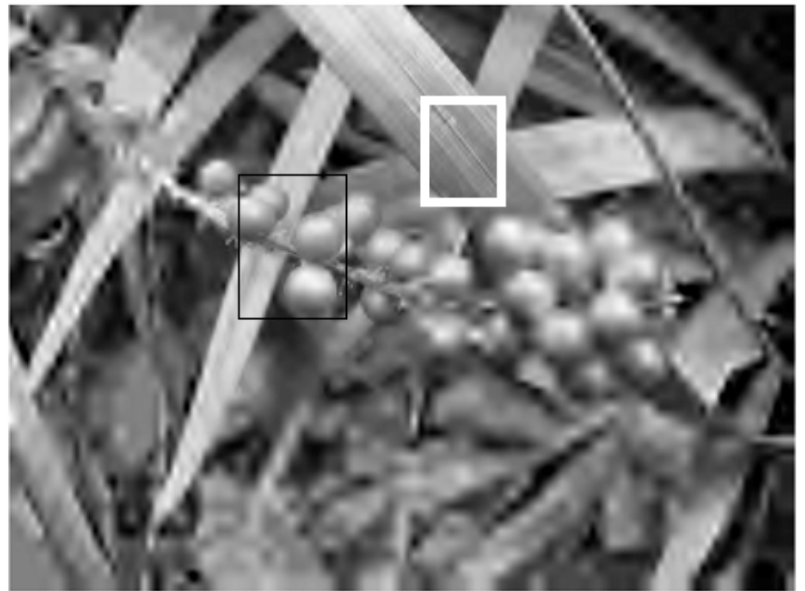

(b)

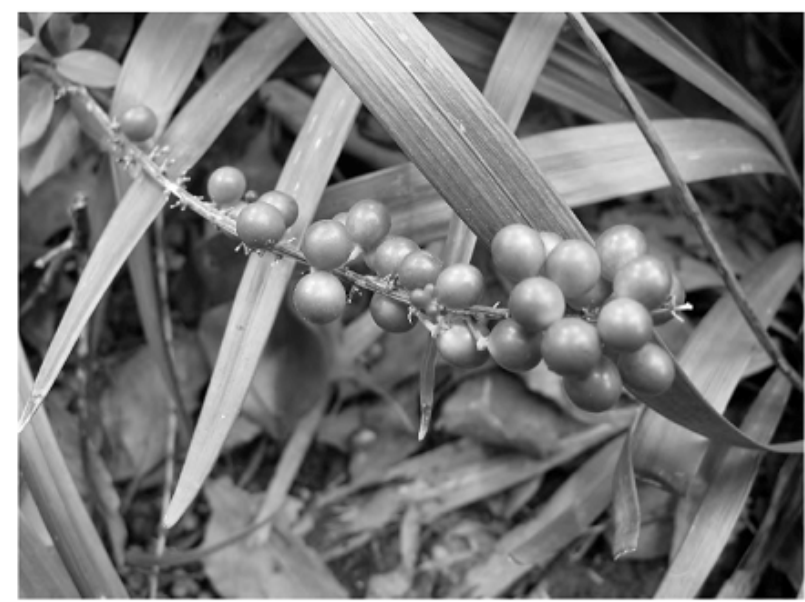

(d)

Fig.3 illustrate of proposed method

[1] JPEG2000 Part I: Final Draft International Standard (ISO/IEC FDIS15444-1).

[2] ISO/IEC JTC 1/SC 29/WG 1 (ITU-T SG8) JPEG 2000 Part II Final Committee, Draft, Dec. 2000.

[3] C.Christopoulos, J.Akelf, and M. Larsson, "Efficient methods for encoding regions of interest in the upcoming JPEG2000 still image coding standard", IEEE Signal Processing lett, vol.7, pp 247-249, sept,2000

[4] Z. Wand and A.C.Bovik,"Bitplane-by-bitplane shift(BbBshift) -A suggestion for JPEG2000 region if interest coding", IEEE Signal Processing Lette, vo19,pp160-162, May 2002

[5] L.Liu and G.Fan,"A new JPEG2000 region of interest image coding method: partial significant bitplanes shift", IEEE Signal Processing Lett, vol.10, pp 35-38, Feb.2003

[6] Victor Sanchez, Anup Basu and Mrinal K. Mandal, "prioritized region of interest coding in JPEG2000", IEEE Trans on ciucuits and systems for video tech, vol 14, pp11491155, Sep, 2004

[7] R.Rosenbaum and H. Schumann, "Flexible, dynamic and compliant region of interest coding in JPEG2000", in Proc .IEEE int. Conf, Image Processing(ICIP), Rochester, New York, Sept,2002, pp101-104
[8] Tammam Tillo, Gabriella Olmo, "A Novel Multiple description coding scheme compatible with the JPEG2000 decoder", IEEE signal processing lett, vol 11,pp 908-911, Nov 2004

[9] David Taubman, Rene Rosenbaum, "rate-distortion optimized interactive browsing of JPEG2000 image ", proceeding of 2003 international conference on image processing ,pp III765-8,vol2R

[10] David Taubman, M. W. Marcellin, JPEG2000: Image Compression Fundamentals, Standards, and Practice, Kluwer Academic Publishers, Boston, 2002.

[11] Nguyen, V. Chandran, S.Sridharan,"important prioritization coding in JPEG2000 for interpretability with applications to surveillance image", SPIE visual communication and image processing, vol 5150,2003, pp806-817 